\title{
Drug interactions: lipoxygenase inhibitors interfere with ropivacaine-induced vasoconstriction
}

\author{
Daisy T. Joo, MD, PhD · Gail K. Wong, MBBS
}

Published online: 27 February 2009

(c) Canadian Anesthesiologists' Society 2009

Local anesthetics are used to facilitate analgesia through their inhibition of sensory nerve conduction. ${ }^{1}$ As sodium channel blockers, ${ }^{1}$ local anesthetics inhibit the propagation of action potentials along nerve axons participating in the transmission of pain signals through the peripheral and central nervous systems. ${ }^{2}$ Aside from sodium channel inhibition, it is now becoming clear that local anesthetics have actions on other important cellular components. In this issue of the Journal, Sung et al. ${ }^{3}$ explore the mechanisms of ropivacaine-induced vasoconstriction through the modulation of arachidonic acid metabolism and production of vasoactive leukotrienes. Their article raises the interesting possibility that existing drug therapies in patients can affect the pharmacokinetics of co-administered local anesthetics.

Ropivacaine is a relatively new, long acting aminoamide local anesthetic that is structurally similar to bupivacaine. However, ropivacaine has relatively low central nervous system (CNS) toxicity and cardiotoxicity compared to bupivicaine. ${ }^{4,5}$ The pharmacokinetic properties of these two local anesthetics are similar with respect to their $\mathrm{pKa}$ values and protein binding properties. ${ }^{5}$

D. T. Joo, MD, PhD ( $ه)$

Department of Anesthesia, Program in Neurosciences and Mental Health, Research Institute, Hospital for Sick Children, 555 University Ave., Rm. 5013D, Toronto, ON M5G 1X8, Canada

e-mail: daisy_joo@yahoo.com

G. K. Wong, MBBS

Department of Anesthesia, Hospital for Sick Children, 555 University Ave., Rm. 2220, Toronto, ON M5G 1X8, Canada

D. T. Joo, MD, PhD · G. K. Wong, MBBS

Department of Anesthesia, University of Toronto,

Toronto, ON, Canada
However, ropivacaine has a smaller volume of distribution, higher plasma clearance ${ }^{5}$ and is less lipid soluble than bupivacaine. Because of its lower lipid solubility and epidural/neural fat absorption, ropivacaine used for epidural and peripheral nerve blockade has a reduced duration of action compared to bupivacaine. The shorter duration of action is due to a greater plasma resorption resulting in a lower elimination half-life $\left(\mathrm{t}_{1 / 2}\right)$ and higher maximal concentration in the plasma $\left(\mathrm{C}_{\max }\right) \cdot{ }^{6-9}$ In contrast, local wound infiltration of ropivacaine results in a longer duration of action compared to bupivacaine, which has been attributed to a direct vasoconstrictive effect of ropivacaine on surrounding vessels. ${ }^{10,11}$

In 2005, Yu et al. ${ }^{12}$ demonstrated the direct vasoconstrictive effect of ropivacaine on de-endothelialized rat aortic vasculature which Sung et al. ${ }^{3}$ reaffirm in this issue. Sung et al. ${ }^{3}$ demonstrated that clinically relevant concentrations of S-ropivacaine (Naropin ${ }^{\mathrm{TM}}$ ) at between $10 \mu \mathrm{M}$ to $1 \mathrm{mM}$ caused vasoconstriction of de-endothelialized rat aorta. These concentrations, though much above central neurotoxic and cardiotoxic thresholds, would be found in proximity of nerves during peripheral, neuraxial, or local injections of ropivacaine. The direct vasomotor actions of ropivacaine have been studied in different vascular structures including capillaries, ${ }^{10}$ femoral veins, ${ }^{11}$ umbilical arteries, ${ }^{13}$ femoral arteries, ${ }^{11}$ and the aorta. ${ }^{12,14,15}$ Some of these studies showed no effect of ropivacaine on impairment of vasorelaxation ${ }^{15}$ while others observed its direct vasoconstrictive effects. ${ }^{12,14}$ The vasoconstrictive properties of ropivacaine are not unique, and are shared by several other amide local anesthetics such as lidocaine ${ }^{16-18}$ and bupivacaine. ${ }^{15}$ Unfortunately, the preparations used by both Sung et al. ${ }^{3}$ and $\mathrm{Yu}$ et al. ${ }^{12}$ were de-endothelialized, thereby, eliminating the effect of nitric oxide- and some $\mathrm{K}_{\mathrm{ATP}}$ channel-induced vasorelaxation mechanisms that 
may be modulated by local anesthetics. Therefore, the summative effects of these opposing vasoactive properties of ropivacaine in normal subjects and those with endothelial dysfunction, including diabetics and atherosclerotic patients, will require further investigation.

$\mathrm{Yu}$ et al. ${ }^{12}$ showed that protein kinase $\mathrm{C}$ (PKC) and p44/42 mitogen-activated protein kinase (MAPK) phosphorylation and rho kinase membrane translocation occurred in the presence of ropivacaine and that PKC, rho kinase, and p44/42 MAPK inhibition reduced ropivacaineinduced aortic smooth muscle contraction. In a follow-up study from the same laboratory, Tokinaga et al. ${ }^{14}$ demonstrated that L-type $\mathrm{Ca}^{2+}$ channel inhibition by nicardipine and $\mathrm{IP}_{3}$ receptor inhibition by 2 -aminoethoxydiphenyl borate reduced ropivacaine-induced aortic smooth muscle inhibition. Therefore, a common theme is that all these ropivacaine-modulated enzyme and receptor/channel pathways lead to an intracellular rise in $\mathrm{Ca}^{2+}$, thereby, activating smooth muscle contractions responsible for the direct vasoconstriction of the rat aorta.

Sung et al. ${ }^{3}$ also demonstrated that the L-type $\mathrm{Ca}^{2+}$ channel blocker, verapamil, inhibits ropivacaine-induced vasoconstriction. However, their novel and major finding was that the inhibition of lipoxygenase and 5-lipoxygenase (5-LOX) also inhibited ropivacaine-induced vasoconstriction in the de-endothelialized rat aorta. Arachidonyl phospholipids are ubiquitous components of all lipid membranes. The cytosolic enzyme phospholipase $\mathrm{A}_{2}$ hydrolyzes arachidonyl phospholipids to produce arachidonic acid which is further broken down via three separate pathways depending on the enzymes: cyclooxygenase (COX), lipoxygenase, and P450 epoxygenase ${ }^{19}$ (Fig. 1). For each group, isoforms of these enzymes exist, resulting in different products of their activity. 5-LOX further breaks down arachidonic acid to form the eicosanoids: leukotrienes and lipoxins. Leukotrienes are signalling molecules with important functions in immunity, inflammation, and regulation of vascular smooth muscle tone (Fig. 1); effects are produced by a leukotriene-modulated elevation of intracellular $\mathrm{Ca}^{2+} .20$

Sung et al. showed that this vasoactivity was inhibited by the phospholipase $\mathrm{A}_{2}$ inhibitor, quinacrine, and further down the pathway by the lipoxygenase inhibitor, nordihydroguaiaretic acid at $10 \mu \mathrm{M}$, and specifically by the 5-LOX, AA-861 at $10 \mu \mathrm{M}$ (see Arachidonic Acid Metabolism Pathway in the Fig. 1). Despite the authors' conclusions that the $\mathrm{COX}$ inhibitor, indomethacin, also attenuated ropivacaine-induced vasoconstriction, this was found to be at a concentration of indomethacin that also blocked lipoxygenase activity. Therefore, inhibitors of COXs and cytochrome P450 epoxygenases important to other parallel pathways of arachidonic acid metabolism

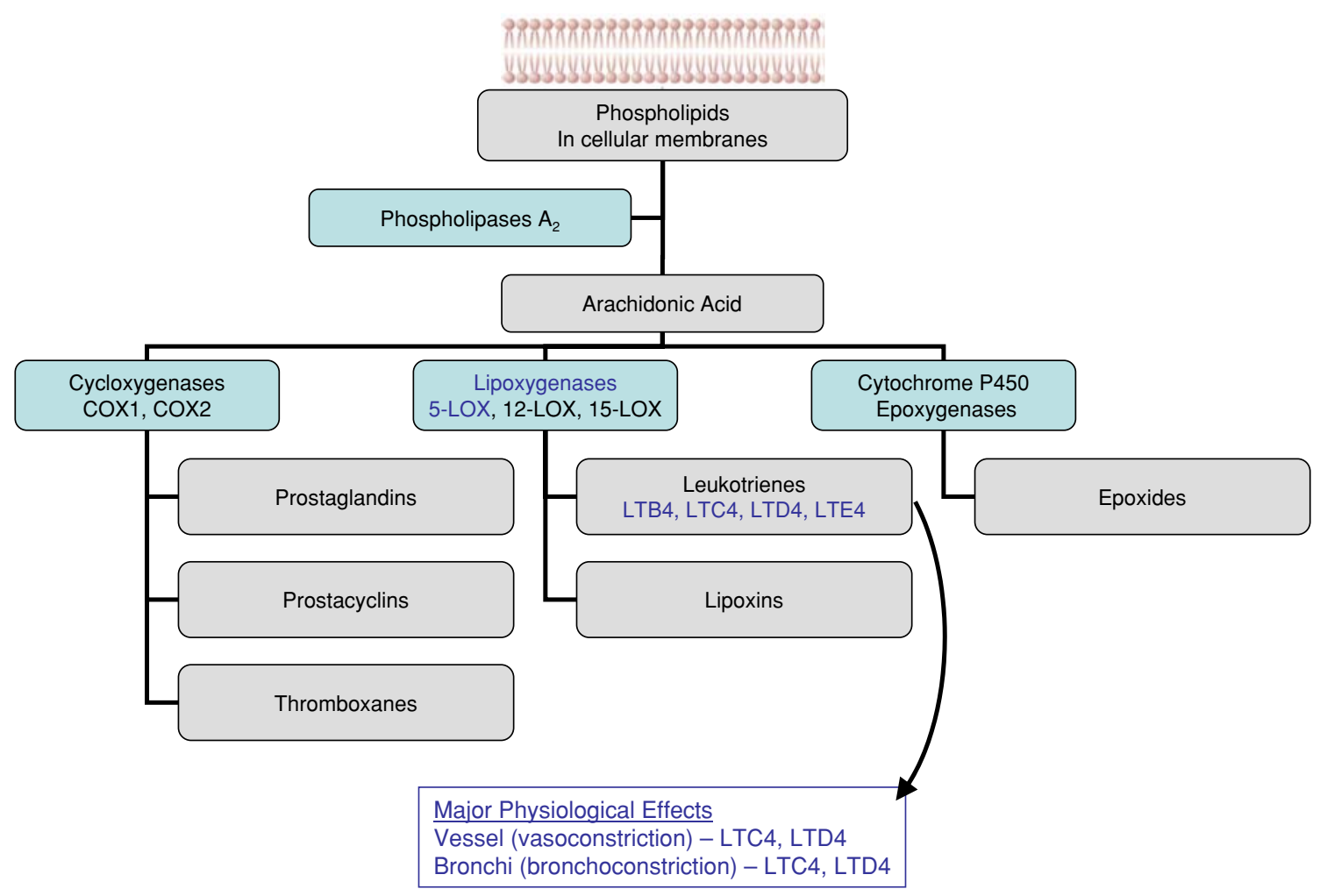

Fig. 1 Arachidonic acid metabolism pathway 
(Fig. 1) are likely ineffective for reducing ropivacaineinduced vasoconstriction. With the relevance of lipoxygenase activity on ropivacaine-induced vasoconstriction, follow-up studies are required to determine whether this is due directly to an increased level of leukotrienes presumably through 5-LOX activity or through the 5-LOX unrelated 5-lipoxygenase activating proteins (FLAPs).

In immunity and inflammatory disease processes, proinflammatory leukotrienes $\mathrm{LTB}_{4}, \mathrm{LTC}_{4}, \mathrm{LTD}_{4}$, and $\mathrm{LTE}_{4}$ are formed by the oxidation of arachidonic acid by 5lipoxygenase (Fig. 1) in leukocytes. These leukotrienes signal $\mathrm{G}$ protein coupled receptors on vascular and bronchial cellular surfaces resulting in vasoconstriction and bronchoconstriction. ${ }^{21}$ Therefore, both leukotriene receptor inhibitors (e.g., montelukast or Singulair ${ }^{\mathrm{TM}}$ ) and 5-LOX inhibition such as with zileutin $\left(\mathrm{Zyflo}^{\mathrm{TM}}\right)$ have been used effectively in asthma. ${ }^{21}$ Leukotrienes have also been implicated in allergic rhinitis. Whether the plasma levels of 5-LOX inhibitors can reach levels at which they impair ropivacaine-induced vasoconstriction is unknown, but may have implications for reducing the duration of ropivacaine local anesthesia.

Leukotrienes can also be elevated by 5-LOX activating FLAPs. Recently, a genotypic association with a FLAP haplotype (ALOX5AP gene variant) has been made in patients manifesting atherosclerotic cardiovascular disease. $^{21}$ This has generated pharmaceutical interest in atherosclerosis reduction therapy using a category of FLAP inhibitors (e.g., indole MK-886 and BAY X1005 for which phase I and II trials are completed) that has previously been shown to reduce leukotriene activity in asthmatics. $^{21}$ If FLAP inhibitors are proven useful for cardiovascular disease, there may be an exponential increase in the patient population on chronic therapy with which ropivacaine may have a shortened duration of action for local anesthesia.

In an era of increasing use of chronic medical therapy and polypharmacy in patients presenting for surgery, the anesthesiologists' continuing education on drug interactions has required rapid and exponential expansion. In this issue of the Journal, the study by Sung et al. ${ }^{3}$ focuses attention on the fact that these interactions are not limited to agents for general anesthesia, but may also affect local anesthetic pharmacology. Perhaps in the future, drug interactions will be so well delineated that anesthetic pharmacokinetic prediction data automatically adjusted for effects from co-administered pharmaceuticals can be readily available for each patient under treatment. However, before that becomes a reality, ongoing research which further elucidates the sites of actions of anesthetic drugs and their interactions through common pathways and receptor sites, and translation of this knowledge to practice, are crucial to the advancement of anesthetic care.

\section{Interactions médicamenteuses: les inhibiteurs de la lipoxygénase interfèrent avec la vasoconstriction provoquée par la ropivacaïne}

Les anesthésiques locaux sont utilisés pour faciliter l'analgésie grâce à leur inhibition de la conduction des nerfs sensitifs. ${ }^{1}$ En tant que bloquants des canaux sodiques, ${ }^{1}$ les anesthésiques locaux inhibent la propagation des potentiels d'action le long des axones qui participent à la transmission des signaux douloureux à travers les systèmes nerveux central et périphérique. ${ }^{2}$ Outre leurs propriétés inhibitrices des canaux sodiques, il est désormais clair que les anesthésiques locaux agissent aussi sur d'importantes composantes cellulaires. Dans ce numéro du Journal, Sung et coll. ${ }^{3}$ explorent les mécanismes de la vasoconstriction provoquée par la ropivacaïne qui modifie le métabolisme de l'acide arachidonique et la production de leucotriènes vasoactifs. Leur article avance la possibilité intéressante que les traitements médicamenteux existants pourraient avoir un effet sur la pharmacocinétique des anesthésiques locaux co-administrés.

La ropivacaïne est un anesthésique local à action prolongée de la famille des amino-amides relativement nouveau et présente une structure semblable à celle de la bupivacaïne. Cependant, la ropivacaïne possède relativement peu de toxicité pour le système nerveux central (SNC) et de cardiotoxicité par rapport à la bupivacaïne. ${ }^{4,5}$ Les propriétés pharmacocinétiques de ces deux anesthésiques locaux sont semblables en ce qui a trait à leurs valeurs de $\mathrm{pKa}$ et à leurs propriétés de liaison aux protéines. ${ }^{5}$ Néanmoins, la ropivacaïne présente un plus petit volume de distribution, une clairance plasmatique plus élevée et est moins liposoluble que la bupivacaïne. En raison de sa solubilité lipidique et de son absorption réduite dans les graisses, lorsqu'elle est utilisée pour réaliser des péridurales ou des blocs du nerf périphérique, la durée d'action de la ropivacaïne est réduite comparativement à celle de la bupivacaïne. Cette durée d'action plus courte est causée par une résorption plasmatique plus importante liée à sa demi-vie d'élimination plus basse $\left(\mathrm{t}_{1 / 2}\right)$ et à une concentration maximale plus élevée dans le plasma $\left(\mathrm{C}_{\max }\right){ }^{6-9}$ En revanche, une infiltration locale de ropivacaïne dans une blessure a une durée d'action plus longue que la bupivacaïne, ce qui a été attribué à un effet vasoconstricteur direct de la ropivacaïne sur les vaisseaux environnants. ${ }^{10,11}$

En 2005, Yu et coll. ${ }^{12}$ ont démontré l'effet vasoconstricteur direct de la ropivacaïne chez le rat sur la vasculature aortique de laquelle on avait ôté l'endothélium, effet que Sung et coll. ${ }^{3}$ confirment dans ce numéro. Sung et coll. ${ }^{3}$ ont démontré que des concentrations pertinentes d'un 
point de vue clinique de S-ropivacaïne (Naropin ${ }^{\mathrm{TM}}$ ) situées entre $10 \mu \mathrm{M}$ et $1 \mathrm{mM}$ provoquaient une vasoconstriction de l'aorte sans endothélium chez le rat. Ces concentrations, quoique bien plus élevées que les seuils cardiotoxiques et neurotoxiques pour le $\mathrm{SNC}$, se trouvaient à proximité des nerfs pendant des injections périphériques, neuraxiales ou locales de ropivacaïne. Les actions vasomotrices directes de la ropivacaïne ont été étudiées dans différentes structures vasculaires, notamment les capillaires, ${ }^{10}$ les veines fémorales, ${ }^{11}$ les artères ombilicales ${ }^{13}$ les artères fémorales, ${ }^{11}$ et l'aorte. ${ }^{12,14,15}$ Certaines études n'ont pas montré d'effet de la ropivacaïne sur la détérioration de la vasorelaxation, ${ }^{15}$ alors que d'autres ont observé des effets vasoconstricteurs directs. ${ }^{12,14}$ La ropivacaïne n'est pas le seul anesthésique local à avoir des propriétés vasoconstrictrices; en effet, ces propriétés sont partagées par plusieurs autres anesthésiques locaux de la famille des amides, tels que la lidocaïne ${ }^{16-18}$ et la bupivacaïne. ${ }^{15}$ Malheureusement, les préparations utilisées par Sung et coll. $^{3}$ et $\mathrm{Yu}$ et coll. ${ }^{12}$ ont éliminé l'effet de certains des mécanismes de vasorelaxation provoqués par l'acide nitrique et les canaux $\mathrm{K}_{\mathrm{ATP}}$, lesquels pourraient être modulés par les anesthésiques locaux par dé-endothélialisation. Par conséquent, les effets cumulatifs des propriétés vasoactives opposées de la ropivacaïne chez les patients normaux et ceux présentant une dysfonction endothéliale, comme par exemple les patients diabétiques ou athéroscléreux, nécessitent des recherches plus approfondies.

$\mathrm{Yu}$ et coll. ${ }^{12}$ ont démontré que la phosphorylation de la protéine kinase $\mathrm{C}(\mathrm{PKC})$ et de la protéine kinase activée par les mitogènes p44/42 (MAPK) ainsi que la translocation de la membrane rho kinase survenaient en présence de ropivacaïne et que l'inhibition de la PKC, la rho kinase et la MAPK p44/42 réduisait la contraction du muscle lisse de l'aorte provoquée par la ropivacaïne. Dans une étude de suivi provenant du même laboratoire, Tokinaga et coll. ${ }^{14}$ ont démontré que l'inhibition des canaux $\mathrm{Ca}^{2+}$ de type $\mathrm{L}$ par la nicardipine et l'inhibition des récepteurs IP3 par le 2-aminoethoxydiphenyl borate réduisaient l'inhibition de la contraction du muscle lisse de l'aorte causée par la ropivacaïne. Ainsi, un thème récurrent est que ces différentes voies enzymatiques et ces récepteurs/canaux modulés par la ropivacaïne engendrent une augmentation de $\mathrm{Ca}^{2+}$ intracellulaire, activant par-là même les contractions du muscle lisse qui sont responsables de la vasoconstriction directe de l'aorte du rat.

Sung et coll. ${ }^{3}$ ont également démontré qu'un bloquant du canal $\mathrm{Ca}^{2+}$ de type $\mathrm{L}$, le vérapamil, inhibe la vasoconstriction provoquée par la ropivacaïne. Toutefois, leur découverte la plus importante était que l'inhibition de la lipoxygénase et la 5-lipoxygénase (5-LOX) inhibait à son tour la vasoconstriction provoquée par ropivacaïne de l'aorte dé-endothélialisée chez le rat. Les phospholipides arachidonyl sont des composants omniprésents de toutes les membranes lipidiques. L'enzyme phospholipase $\mathrm{A}_{2}$ cytosolique hydrolyse les phospholipides arachidonyl afin de produire de l'acide arachidonique, lequel est ensuite fractionné par trois voies différentes selon les enzymes: la cyclooxygénase (COX), la lipoxygénase et l'époxygénase $\mathrm{P} 450^{19}$ (voir figure). Dans chaque groupe, des isoformes de ces enzymes existent, dont l'activité crée divers produits. La 5-LOX fractionne l'acide arachidonique afin de former les eicosanoïdes- leucotriènes et lipoxines. Les leucotriènes sont des molécules de signalisation qui jouent un rôle important dans les mécanismes d'immunité, d'inflammation, et dans la régulation du tonus du muscle lisse vasculaire (voir figure); les effets produits par un leucotriène ont modulé l'augmentation du $\mathrm{Ca}^{2+}$ intracellulaire. ${ }^{20}$

Dans leur étude, Sung et coll. ont démontré que cette vasoactivité était inhibée par un inhibiteur de la phospholipase $\mathrm{A}_{2}$, la quinacrine, et par un inhibiteur de la voie descendante de la lipoxygénase, l'acide nordihydroguaiarétique à $10 \mu \mathrm{M}$, et plus spécifiquement par la 5-LOX, AA-861 à $10 \mu \mathrm{M}$ (voir la voie métabolique de l'acide arachidonique dans la figure). En dépit des conclusions des auteurs selon lesquelles l'indométhacine, un inhibiteur de la COX, atténuait également la vasoconstriction provoquée par la ropivacaïne, il a été démontré que cet effet survenait à une concentration d'indométhacine qui inhibait également l'activité de la lipoxygénase. Pour cette raison, les inhibiteurs des COX et les époxygénases du cytochrome P450 qui jouent un rôle important dans d'autres voies parallèles du métabolisme de l'acide arachidonique (voir figure) n'ont probablement pas d'effet de réduction sur la vasoconstriction provoquée par la ropivacaïne. Étant donné la pertinence de l'activité de la lipoxygénase sur la vasoconstriction provoquée par la ropivacaïne, d'autres études de suivi devraient être réalisées afin de déterminer si cet effet est dû directement à un niveau plus élevé de leucotriènes, vraisemblablement en raison de l'activité de la 5-LOX ou des protéines d'activation de la 5-lipoxygénase (FLAP) non associées à la 5-LOX.

Dans les processus pathologiques immunitaires et inflammatoires, les leucotriènes pro-inflammatoires $\mathrm{LTB}_{4}$, $\mathrm{LTC}_{4}, \mathrm{LTD}_{4}$, et $\mathrm{LTE}_{4}$ sont formées par l'oxydation de l'acide arachidonique par la 5-lipoxygénase (voir figure) dans les leucocytes. Ces leucotriènes signalent les récepteurs associés à la protéine $\mathrm{G}$ sur les surfaces cellulaires vasculaires et bronchiques, provoquant la vasoconstriction et la bronchoconstriction. ${ }^{21}$ Pour cette raison, des inhibiteurs des récepteurs des leucotriènes (par ex. montelukast ou Singulair ${ }^{\mathrm{TM}}$ ) et l'inhibition de la 5-LOX comme avec la zileutine $\left(\right.$ Zyflo $\left.^{\mathrm{TM}}\right)$ ont été utilisés pour traiter l'asthme. ${ }^{21}$ Les leucotriènes ont également été impliquées dans les rhinites allergiques. Nous ne savons pas si les niveaux plasmatiques des inhibiteurs de la 5-LOX peuvent atteindre 
des seuils auxquels ils bloquent la vasoconstriction provoquée par la ropivacaïne, ce qui pourrait avoir des implications dans la réduction de la durée de l'anesthésie locale avec la ropivacaïne.

Le taux de leucotriène peut également augmenter avec les FLAP non associées à la 5-LOX. Récemment, une association génotypique avec un haplotype de FLAP (variante génétique $A L O X 5 A P$ ) a été observée chez des patients présentant une maladie cardiovasculaire athérosclérotique. ${ }^{21}$ Cette découverte a provoqué un intérêt pharmaceutique pour des traitements de réduction de l'athérosclérose utilisant une catégorie d'inhibiteurs de la FLAP (par ex. indole MK-886 et BAY X1005, pour lesquels les essais de phase I et II ont été complétés), et pour lesquels il avait été précédemment démontré qu'ils réduisaient l'activité des leucotriènes chez les patients asthmatiques. ${ }^{21}$ Si l'on parvient à démontrer que les inhibiteurs de FLAP sont utiles pour le traitement des maladies cardiovasculaires, il est possible qu'on assiste à une augmentation exponentielle de la population de patients traitée de façon chronique et pour laquelle la ropivacaïne aurait une durée d'action plus courte lorsqu'elle est utilisée pour réaliser une anesthésie locale.

À une époque où l'on recourt de plus en plus aux traitements médicaux chroniques et à la polypharmacie chez les patients devant subir une chirurgie, la formation continue des anesthésiologistes quant aux interactions médicamenteuses a due être étendue de façon rapide et exponentielle. Dans ce numéro du Journal, l'étude de Sung et coll. ${ }^{3}$ souligne le fait que ces interactions ne se limitent pas aux agents utilisés pour l'anesthésie générale, mais pourraient également affecter la pharmacologie des anesthésiques locaux. Peut-être qu'à l'avenir les interactions médicamenteuses seront tellement bien décrites que les données de prédiction de la pharmacocinétique anesthésiques, automatiquement ajustées pour tenir compte des effets des médicaments co-administrés, seront facilement accessibles pour chaque patient traité. Cependant, avant que cela ne devienne une réalité, des recherches approfondies afin d'élucider les sites d'action des agents anesthésiques et leurs interactions par des voies courantes et des sites de récepteurs, ainsi que la transposition de ces connaissances en pratique, sont essentielles afin de faire avancer les soins anesthésiques.

Competing interests None declared.

\section{References}

1. Yanagidate F, Strichartz GR. Local anesthetics. Handb Exp Pharmacol 2007; 177: 95-127.

2. Butterworth JF 4th, Strichartz GR. Molecular mechanisms of local anesthesia: a review. Anesthesiology 1990; 72: 711-34.
3. Sung HJ, Sohn JT, Park JY, Hwang EM, Baik JS, Ogawa K. Direct effect of ropivacaine involves lipoxygenase pathway activation in rat aortic smooth muscle. Can J Anesth 2009; 56: 4.

4. Akerman B, Hellberg IB, Trossvik $C$. Primary evaluation of the local anaesthetic properties of the amino amide agent ropivacaine (LEA 103). Acta Anaesthesiol Scand 1988; 32: 571-8.

5. Scott DB, Lee A, Fagan D, Bowler GM, Bloomfield P, Lundh R. Acute toxicity of ropivacaine compared with that of bupivacaine. Anesth Analg 1989; 69: 563-9.

6. Morrison LM, Emanuelsson BM, McClure JH, et al. Efficacy and kinetics of extradural ropivacaine: comparison with bupivacaine. Br J Anaesth 1994; 72: 164-9.

7. Irestedt L, Ekblom A, Olofsson C, Dahlstrom AC, Emanuelsson $B M$. Pharmacokinetics and clinical effect during continuous epidural infusion with ropivacaine $2.5 \mathrm{mg} / \mathrm{ml}$ or bupivacaine $2.5 \mathrm{mg} / \mathrm{ml}$ for labour pain relief. Acta Anaesthesiol Scand 1998; 42: 890-6.

8. Sandler AN, Arlander E, Finucane BT, et al. Pharmacokinetics of three doses of epidural ropivacaine during hysterectomy and comparison with bupivacaine. Can J Anaesth 1998; 45: 843-9.

9. Kopacz DJ, Emanuelsson BM, Thompson GE, Carpenter RL, Stephenson $C A$. Pharmacokinetics of ropivacaine and bupivacaine for bilateral intercostal blockade in healthy male volunteers. Anesthesiology 1994; 81: 1139-48.

10. Kopacz DJ, Carpenter RL, Mackey DC. Effect of ropivacaine on cutaneous capillary blood flow in pigs. Anesthesiology 1989; 71: 69-74.

11. Nakamura K, Toda H, Kakuyama M, et al. Direct vascular effect of ropivacaine in femoral artery and vein of the dog. Acta Anaesthesiol Scand 1993; 37: 269-73.

12. Yu J, Tokinaga Y, Kuriyama T, Uematsu N, Mizumoto K, Hatano $Y$. Involvement of $\mathrm{Ca}^{2+}$ sensitization in ropivacaine-induced contraction of rat aortic smooth muscle. Anesthesiology 2005; 103: 548-55.

13. Bariskaner H, Tuncer S, Taner A, Dogan N. Effects of bupivacaine and ropivacaine on the isolated human umbilical artery. Int J Obstet Anesth 2003; 12: 261-5.

14. Tokinaga Y, Ogawa K, Yu J, Kuriyama T, Minonishi T, Hatano $Y$. Mechanism of the ropivacaine-induced increase in intracellular $\mathrm{Ca}^{2+}$ concentration in rat aortic smooth muscle. Acta Anaesthesiol Scand 2007; 51: 1155-60.

15. Dojo M, Kinoshita H, Nakahata K, Kimoto $Y$, Hatano $Y$. Effects of bupivacaine enantiomers and ropivacaine on vasorelaxation mediated by adenosine triphosphate-sensitive $\mathrm{K}(+)$ channels in the rat aorta. Anesthesiology 2004; 101: 251-4.

16. Kinoshita H, Nakahata $K$, Dojo M, Kimoto $Y$, Hatano $Y$. Lidocaine impairs vasodilation mediated by adenosine triphosphatesensitive $\mathrm{K}+$ channels but not by inward rectifier $\mathrm{K}+$ channels in rat cerebral microvessels. Anesth Analg 2004; 99: 904-9.

17. Kinoshita H, Iranami H, Kimoto Y, Dojo M, Hatano Y. Mild alkalinization and acidification differentially modify the effects of lidocaine or mexiletine on vasorelaxation mediated by ATPsensitive K+ channels. Anesthesiology 2001; 95: 200-6.

18. Kinoshita H, Ishikawa T, Hatano $Y$. Differential effects of lidocaine and mexiletine on relaxations to ATP-sensitive $\mathrm{K}+$ channel openers in rat aortas. Anesthesiology 1999; 90: 1165-70.

19. Harizi H, Corcuff JB, Gualde N. Arachidonic-acid-derived eicosanoids: roles in biology and immunopathology. Trends Mol Med 2008; 14: 461-9.

20. Rovati GE, Capra V. Cysteinyl-leukotriene receptors and cellular signals. Sci World J 2007; 7: 1375-92.

21. Evans JF, Ferguson AD, Mosley RT, Hutchinson JH. What's all the FLAP about?: 5-lipoxygenase-activating protein inhibitors for inflammatory diseases. Trends Pharmacol Sci 2008; 29: 72-8. 